\title{
Theory into practice: opportunities \& applications of quantitative genetics in plants
}

\author{
Alison R. Bentley $\mathbb{D}^{1} \cdot$ Lindsey J. Compton $\mathbb{( \mathbb { D }}^{2}$
}

Received: 11 September 2020 / Accepted: 13 September 2020

(c) The Genetics Society 2020

Quantitative genetics seeks to understand the complex network of genes and the ways they interact, both with each other and with environmental factors, to determine complex organismal phenotypes. There are multiple routes for exploitation and application of the fundamental principles of quantitative genetics, along with a growing resource base spanning germplasm collections to genome sequences. Over recent years, this deployment has become increasingly recognised as an urgent need in order to increase crop productivity and support future food, nutrition, and energy security. In this special issue, we focus on plant quantitative genetics: from theory into practice, a theme that formed the focus of the Genetics Society sponsored 2019 UK Plant Quant conference at the University of Birmingham (cosupported by KWS UK Ltd, Elsoms Ackermann Barley, LGC Biosearch Technologies, Limagrain, RAGT Seeds, Syngenta, Branston, Tozer Seeds and Oxford University Press). We present a collection of reviews and original articles that demonstrate the multi-faceted and potential downstream uses of plant quantitative genetics, which should ignite interest within a broad readership, from plant (and animal) geneticists, evolutionary and developmental biologists to breeders.

Bernardo (2020) leads the reader on a thought-provoking journey through 150 years of the study of continuous trait variation, inspiring deep reconsideration of one's own understanding of the field and its current needs. Exploring the challenge of "keeping quantitative genetics current", this Opinion article presents a compelling argument for the

Alison R. Bentley

alison.bentley@niab.com

$\triangle$ Lindsey J. Compton

1.j.compton@bham.ac.uk

1 The John Bingham Laboratory, NIAB, Cambridge CB3 OLE, UK

2 School of Biosciences, University of Birmingham, Edgbaston, Birmingham B15 2TT, UK retention of useful established concepts, retirement of the archaic, support for new methods and borrowing concepts that may be useful from other fields. As the evolution of plant breeding merges the traditional Mendelian perspective tagging traits of interest to a biometrical approach in which genetic relationships are incorporated, the concept of borrowing will become more pressing. The unprecedented scale of current data and technology generation calls for new ways to assess reliability and to simulate the practical and logistic impacts of methodology changes across the entirety of a plant breeding programme.

New concepts and methods are presented and reviewed in several papers in this special issue, including the use of multi-parent populations (Scott et al. 2020), genomic prediction (de Bem Oliveira et al. 2020) and integrated genomic tools (Ali and Borrill 2020). Scott et al. (2020) review crop multi-parent populations as an enduring resource for quantitative genetics research and trait mapping. They also demonstrate the potential value of these experimental populations for use in breeding as a germplasm resource, which may be enhanced by the availability of a companion package of genetic and phenotypic information. In future, this package could include a genomic prediction function to provide material meeting specific trait or genetic requirements. De Bem Oliveira et al. (2020) explore the components for optimal implementation of genomic prediction in autotetraploid blueberry. Evaluating training population size and composition along with marker density and sequencing depth on phenotypic predictions will help to guide decisions on resource allocation, with relevance for genomic prediction in other diploid or polyploid crop species. In the area of novel traits, Ali and Borrill (2020) present the potential of biofortification to address currently low levels of micronutrients in wheat grain and the opportunities afforded by a wealth of recently available genomics tools. Spanning genome assemblies, expression atlases and characterised mutant populations, they describe the pathway to discover and validate novel exploitable variation. They also highlight the importance of global collaboration beyond the lab to 
ensure that the promised benefits to human health can be delivered. Taken together, the suite of new tools presented demonstrate a strong foundational resource of germplasm, trait prediction and integrated 'omics resources founded in genetic principles and available for use in applied crop improvement.

The challenge of adapting crops to changing climates is discussed for wheat (Hyles et al. 2020) and the perennial tree species Populus trichocarpa (Richards et al. 2020). Both argue that there is much value in characterising component genetic controllers of the seasonal timing of plant lifecycles, given its central importance in seasonal adaptation and for optimising crop production in a changing climate. Hyles et al. (2020) review current knowledge on the genetic control of phenology and developmental traits that adapt the major food crop wheat to the diverse global environments in which it is grown. Understanding the underpinning genetics and accurately characterising corresponding phenotypes is central to future breeding efforts. Richards et al. (2020) describe adaptive phenology in the perennial tree species $P$. trichocarpa, incorporating the pressing need to predict and track environmental change, particularly in the breeding and selection of perennial species. Supporting this, they determine the quantitative genetic basis of a number of phenology traits, demonstrating a consistent year-to-year response to seasonal cues. They also report that genetic adaptation to changing seasons is likely, given the detected variation in the species and that this is likely to also apply for phenology shifts in other perennial plant species.

Without geneticists, there would be no field of genetics. This is of course true of all disciplines; however, a decline in engagement in plant science, and science in general, will negatively impact the future talent base. Hale (2020) highlights how secondary school students in areas with low visibility of scientific careers have low engagement in science subjects. This has downstream implications for choice of career paths. Hands-on genetics research projects can be an effective way to engage students, develop personal and scientific skills, and ultimately to change lives by raising aspirations. Hale describes a student-led research project sequencing the chloroplast genomes from daffodils, offering an insight into opportunities for inspiring and engaging the next generation of plant scientists.

Quantitative genetics is a long-established field of research and application, which has always and will con- tinue to contribute a fundamental underpinning for plant breeding. In this special issue, we bring together a range of perspectives, reviews and examples to showcase the breadth of the discipline. Evolution of the field is essential, as is the retention of useful components. A wide range of new resources provide the basis for future research discoveries and genetic gains in breeding. Climate change adaptation will become more pressing, and the speed at which crops can be adapted will determine the ability of the primary production sector to mitigate impacts. Finally, new knowledge must be generated to continue to drive progress, and to that end, the next generation of quantitative geneticists and plant scientists must be inspired, nurtured and allowed to grow.

\section{Compliance with ethical standards}

Conflict of interest The authors declare that they have no conflict of interest.

Publisher's note Springer Nature remains neutral with regard to jurisdictional claims in published maps and institutional affiliations.

\section{References}

Ali MW, Borrill P (2020) Applying genomic resources to accelerate wheat biofortification. Heredity. https://doi.org/10.1038/s41437020-0326-8

Bernardo R (2020) Reinventing quantitative genetics for plant breeding: something old, something new, something borrowed, something BLUE. Heredity. https://doi.org/10.1038/s41437-0200312-1

de Bem Oliveira I, Amadeu RR, Ferrão LFV, Muñoz PR (2020) Optimizing whole-genomic prediction for autotetraploid blueberry breeding. Heredity. https://doi.org/10.1038/s41437-020-00357-x

Hale J (2020) Engaging the next generation of Plant Geneticists through sustained research: an overview of a post-16 project. Heredity. https://doi.org/10.1038/s41437-020-00370-0

Hyles J, Bloomfield MT, Hunt JR, Trethowan RM, Trevaskis B (2020) Phenology and related traits for wheat adaptation. Heredity. https://doi.org/10.1038/s41437-020-0320-1

Richards TJ, Karacic A, Apuli R-P, Weih M, Ingvarsson PK, Rönnberg-Wästljung AC, et al. (2020) Quantitative genetic architecture of adaptive phenology traits in the deciduous tree, Populus trichocarpa (Torr. and Gray). Heredity. https://doi.org/ 10.1038/s41437-020-00363-Z

Scott MF, Ladejobi O, Amer S, Bentley AR, Biernaskie J, et al. (2020) Multi-parent populations in crops: a toolbox integrating genomics and genetic mapping with breeding. Heredity. https://doi.org/10. 1038/s41437-020-0336-6 